\title{
Damping force in the transit-time method of optical stochastic cooling
}

\author{
Alexander Zholents \\ Argonne National Laboratory, 9700 S. Cass Avenue, Argonne, Illinois 60439, USA
}

(Received 27 May 2011; published 12 March 2012)

\begin{abstract}
This brief note clarifies one specific aspect of a transient-time method of optical stochastic cooling that often creates confusion for the first time surveyor of this cooling technique.

DOI: 10.1103/PhysRevSTAB.15.032801

PACS numbers: 41.75. $-\mathrm{i}, 41.60 .-\mathrm{m}, 41.90 .+\mathrm{e}$
\end{abstract}

A cooling system of a transient-time method of optical stochastic cooling uses signal undulator, bypass, optical amplifier, and a kicker undulator as shown in Fig. 1. A detailed description of the method is given in Ref. [1] and a discussion of many practical issues can be found in Ref. [2]. This note focuses only on one specific aspect of the method that is not clearly articulated in $[1,2]$. This missing part often creates confusion for the first time surveyor of this cooling technique.

The source of the confusion is that the damping force in the transit-time method of optical stochastic cooling is not linear and is best characterized by an equation:

$$
\delta_{i}^{(2)}=\delta_{i}^{(1)}-G \sin \left(k R_{56} \delta_{i}^{(1)}\right) .
$$

Here $\delta_{i}^{(1)}$ is a particle relative energy before entering a cooling insertion and $\delta_{i}^{(2)}$ is the particle relative energy after the cooling insertion, $k=2 \pi / \lambda$ is the wave vector and $\lambda$ is the optical wave length, $R_{56}$ is the time-of-flight characteristics of the bypass, and $G$ is a coefficient that is approximately constant to within $3 \%$ for $\left|k R_{56} \delta_{i}^{(1)}\right| \leq 4 \pi$. For simplicity only cooling of the beam energy spread is considered now. According to [1], a particle produces a signal in a signal undulator, then follows the bypass and arrives at the entrance of the kicker undulator in front of its own amplified signal. Usually, the amplifier has a narrower bandwidth than a signal whose relative bandwidth is defined by the inverse number of undulator periods. It is also beneficial to have the same number of undulator periods in the kicker undulator as a number of wave cycles in the amplified signal. But typically it is shorter for practical reasons. In this case the bypass path length is adjusted in such way that particles interact in the kicker undulator with the middle part of their own amplified signals arriving at the entrance of the kicker undulator behind the beginning of the signal waves, slipping behind the waves in the undulator, but still exiting before they end. Moreover, the bypass path length is fine-tuned to ensure that the particle with equilibrium energy arrives exactly at the zero crossing

Published by the American Physical Society under the terms of the Creative Commons Attribution 3.0 License. Further distribution of this work must maintain attribution to the author(s) and the published article's title, journal citation, and DOI. of the wave as shown in Fig. 1 and arrival times of other particles are spread around it. The peculiarity of the damping force discussed here consists in the following. When $\left|k R_{56} \delta_{i}^{(1)}\right| \ll 1$, the damping force in (1) is perfectly linear, but when $\left|k R_{56} \delta_{i}^{(1)}\right|>\pi / 2$, the damping force even changes the sign and appears as the excitation force. Thus, it was found in $[1,2]$ that, in the case of a Gaussian distribution in $\delta$ with a given rms value $\sigma_{\delta}$, the optimal choice for $R_{56}$ is defined by a condition:

$$
R_{56}=1 /\left(k \sigma_{\delta}\right) .
$$

For a given $G$ this condition ensures the fastest rate of cooling and preserves efficient cooling for a majority of the particles in the beam. Evidently, if cooling results in decreasing the value of $\sigma_{\delta}$ with time, then $R_{56}$ should increase with time to keep cooling at the fastest rate. However, $R_{56}$ should be kept constant if $\sigma_{\delta}$ does not change, e.g., when the rate of cooling is balanced by the rate of diffusion. For example, this happens when cooling advances to a point where intrabeam scattering creates a rate of diffusion equal to the rate of cooling.

It was understood, but was not explicitly pointed out in $[1,2]$, that condition (2) has some ambiguity. First, it is only true for a Gaussian distribution. Other beam distributions will end up having a slightly different optimum $R_{56}$ that can be found following the same analysis as in $[1,2]$. Second, condition (2) should be modified if one wants to cool particles located very far in the tails of the distribution because they may be pushed out by the antidamping (excitation) force mentioned above. Actually, it is not immediately evident what happens to the particles in the far tails as cooling of the beam core progresses. The delicate part consists of the following. Even if the particle has large amplitude of oscillations and, thus, spends a large fraction

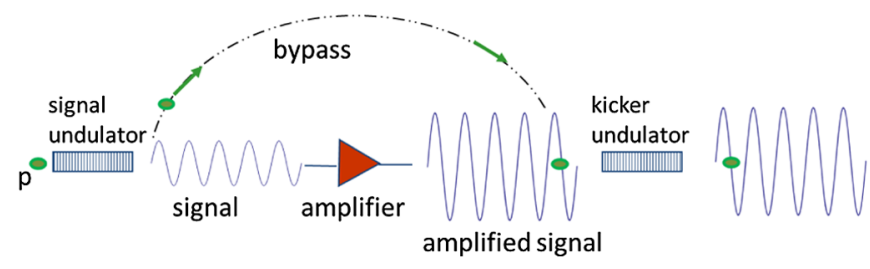

FIG. 1. A cooling system of a transient-time method of optical stochastic cooling [1]. 
of a time in the tails where it experiences antidamping, it also spends some time near the center of the distribution where it experiences damping. Thus, the question is: what prevails, damping or antidamping?

This paper, actually, addresses this question by considering harmonic oscillations affected by the damping force described by Eq. (1). For simplicity, it is also assumed that there is no diffusion and, thus, all particles are expected to go either to a zero point in the case of damping or somewhere else in the case of antidamping. In accordance with the above discussion, different damping behavior of particles is expected depending on the initial value of $\delta=\delta_{0}$.

For a reason that will become clear later, it is more convenient to present the result in units of the initial argument of the sine in Eq. (1), e.g., $\varphi_{0}=k R_{56} \delta_{0}$. What was found is that in the range of $\delta_{0}$ corresponding to $\left|\varphi_{0}\right| \leq$ $1.2 \pi$, all particles go to a zero value. In the range of $\delta_{0}$ corresponding to the range $1.2 \pi \leq\left|\varphi_{0}\right| \leq 3.2 \pi$, all particles are attracted to a new fixed point and continue to oscillate with the amplitude $\delta_{1}$ corresponding to $\left|\varphi_{1}\right|=$ $2 \pi$. In the range of $\delta_{0}$ corresponding to the range $3.2 \pi<$ $\left|\varphi_{0}\right| \leq 5.2 \pi$, all particles are attracted to a different fixed point where they continue to oscillate with the amplitude $\delta_{2}$ corresponding to $\left|\varphi_{2}\right|=4 \pi$. All these regions are shown with different colors in Fig. 2.

It was concluded based on these findings that condition (2) ensures damping to all particles with initial amplitudes up to $\delta_{0} \approx 3.75 \sigma_{\delta}$. All particles with $\delta_{0}>3.75 \sigma_{\delta}$ will eventually be attracted to a new fixed point and then will continue to oscillate with the amplitude $\delta_{1} \approx 2 \pi \sigma_{\delta}$ and so on. Thus, if one wants to cool particles as far in the tail of

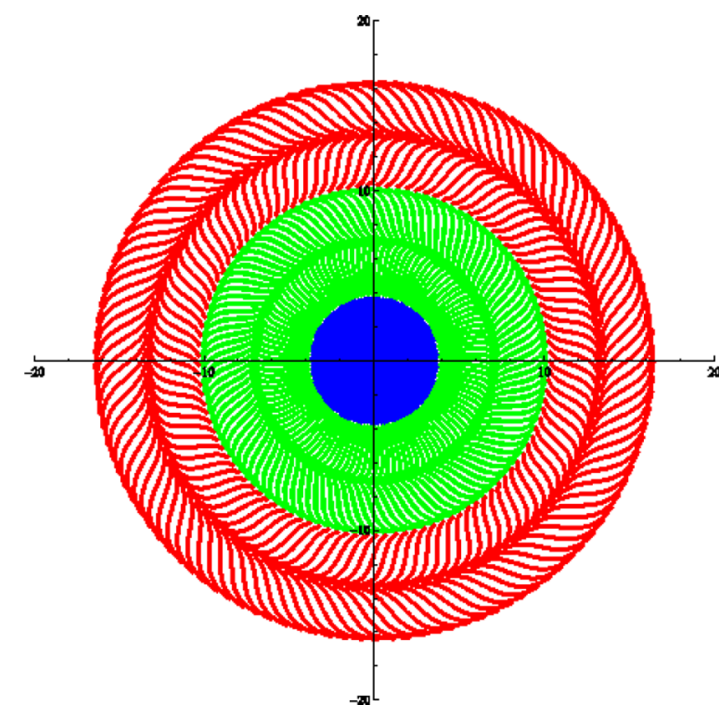

FIG. 2. The phase space portrait of the longitudinal oscillations showing evolution of the particle trajectories (in normalized coordinates) starting from different initial conditions. Different colors correspond to regions with different fixed points (or circles) with values of $\varphi=0,2 \pi$, and $4 \pi$. the distribution as $\delta_{0}=n \sigma_{\delta}$, then condition (2) should be modified to

$$
R_{56}=\frac{3.75}{n k \sigma_{\delta}}
$$

This could be important if there is a mechanism that launches particles from the beam core to large amplitudes like, for example, Touschek scattering or particle scattering on molecules of a residue gas.

So far, only energy cooling was considered, but in $[1,2]$ a simultaneous cooling of the beam emittance and energy was proposed. The analog of condition (2) in this case is a condition:

$$
k \sigma_{\Delta \ell}=1,
$$

where $\sigma_{\Delta \ell}$ is the rms value of the time-of-flight delay $\Delta \ell$ that also accounts for particle coordinates and angles along with the energy spread, e.g., $\Delta \ell=R_{51} x+R_{52} x^{\prime}+R_{56} \delta$. In this case, the analog of the cooling boundaries discussed above is the multidimensional surfaces in the 6D phase space satisfying a condition $k \Delta \ell=1.2 \pi$. Similarly, the circles of a "fixed attraction" will transform into a set of surfaces that satisfy a set of conditions $k \Delta \ell=2 \pi$ or $4 \pi$.

In summary, the choice for the time-of-flight delay in the transit-time method of optical stochastic cooling plays an important role in the optimization of the cooling rate and the area in the $6 \mathrm{D}$ phase space accessible by cooling. One can trade the cooling rate for the increased area and vice versa. The proposed choice in $[1,2]$ ensures the fastest rate of cooling but only for particles located within the area of the phase space containing approximately $99 \%$ of all particles (assuming Gaussian distribution in all coordinates and energy). However, if rare scattering events launch particles beyond this area, then these particles will be attracted to other than zero centers and will continue to stay at large amplitudes. This could affect the beam lifetime and, possibly, create excessive background if such beam is going to be used in the experiment. If this is not acceptable, then one would need to reduce the cooling rate by adjusting the time-of-flight delays and, therefore, increase the $6 \mathrm{D}$ phase space area accessible by cooling.

\section{ACKNOWLEDGMENTS}

I am grateful to Valery Lebedev who prompted these studies pointing out that condition (2) may not be the optimal one in all cases. This work was supported by U.S. DOE under Contract No. DE-AC02-06CH11357.

[1] M. Zolotorev and A. Zholents, Phys. Rev. E 50, 3087 (1994).

[2] M. Babzien, I. Ben-Zvi, I. Pavlishin, I. Pogorelsky, V. Yakimenko, A. Zholents, and M. Zolotorev, Nucl. Instrum. Methods Phys. Res., Sect. A 532, 345 (2004). 